
\title{
28 Research Square \\ The Protective Mechanism of SIRT1 on Cartilage Through Regulation of LEF-1
}

\section{Xueyu Hu}

Ningxia Medical University

Gangning Feng

Ningxia Medical University

\section{Zhiqiang Meng}

Ningxia Medical University

\section{Long Ma}

Ningxia Medical University

Qunhua Jin ( $\square$ jinqunhua2020@163.com )

The General Hospital Of Ningxia Medical University https://orcid.org/0000-0001-7616-3357

\section{Research article}

Keywords: Osteoarthritis, SIRT-1, LEF1, Cartilage

Posted Date: February 19th, 2021

DOI: https://doi.org/10.21203/rs.3.rs-214497/v1

License: (c) (1) This work is licensed under a Creative Commons Attribution 4.0 International License. Read Full License 


\section{Abstract}

Background: Osteoarthritis as a chronic degenerative disease suppresses middle-aged and elderly people all over the world. In this study, we found the expression changes of lymphocyte enhancer factor 1 (LEF-

1) from human specimens, and then found the regulatory relationship between silent information regulator $1 \llbracket S I R T-1 \rrbracket$ and LEF-1 during osteoarthritis development and its effect on cartilage protection.

Material/Methods: First, changes in the expression of LEF-1 protein were found in fresh specimens of the tibial plateau of patients with osteoarthritis, and then the hypothesis was verified mice model. Mice were randomly divided into three groups, including the normal group, model building, and drug spraying group. Each group is divided into two periods: 2 weeks after modeling and 8 weeks after modeling. Histological staining, immunohistochemistry, immunofluorescence, and qPCR experiments were used to detect the expression of SIRT-1, $\beta$-catenin, LEF-1, matrix metalloproteinase 13(MMP-13) and Collagen II.

Result: LEF-1 increased with the severity of osteoarthritis in human specimens. We observed in animals: when osteoarthritis symptoms worsened, the expression of SIRT-1 and Collagen II gradually decreased, the expression of $\beta$-catenin, LEF-1, and MMP-13 and cartilage destruction gradually increased.

Conclusion: Sirt- 1 plays a protective role in cartilage by reducing the expression of $\beta$ - Catenin, LEF-1 and MMP-13, and increasing the expression of collagen II.

\section{Introduction}

Osteoarthritis (OA) is a chronic degenerative joint disease that affects middle and older aged people worldwide. $\mathrm{OA}$ is mainly caused by loss of articular cartilage, joint soft tissue remodeling, and changes in synovial inflammatory [1]. Progressive damage to articular cartilage is characterized by structural changes. Cartilage is mainly composed of chondrocytes and extracellular matrix, among which chondrocytes account for about $1 \%$ of the total cartilage volume, and the rest of the mass is formed by the extracellular matrix $[2,3]$. The extracellular matrix is rich in collagen fibers and aggrecan, which can nourish, support, and protect the cartilage cells. Various studies have shown that during the prognosis of OA, articular chondrocytes secrete pro-inflammatory cytokines (IL-1 $\beta$, TNF- $\alpha$ ) and matrix decomposing factors such as MMP-13, A Disintegrin And Metalloproteinase With Thrombospondin (ADAMTS) [4]. The increased expression of these proteins aggravates the breakdown of the extracellular matrix and significantly affects the survival of chondrocytes. Thus, it leads to the occurrence and progression of OA pathological processes.

Histone deacetylase (HDAC) can specifically modify chromatin structure and regulate transcription factor activity. It catalyzes the acetyl groups from the histones tails and other proteins to form deacetylated substrates, acetyl groups, and a water molecule[5]. SIRT-1 is a class III HDAC, which has been proposed to inhibit osteoarthritis. Specimens of subchondral osteoblast from OA patients show significant reduction in the expression of the SIRT-1 gene compared to normal adult joint specimens [6]. In the articular cartilage of SIRT-1-deficient mice, chondrocyte apoptosis was increased while the expression of type II 
collagen in the extracellular matrix was significantly decreased. The expression of aggregate MMP-13 was also increased. In addition, it confirmed that the high expression of the SIRT1 gene could halt the inflammatory changes through inhibition of IL-1 $\beta$ in human osteoarticular chondrocytes [7]. However, the specific effect of SIRT-1 on cartilage is still not clear.

LEF-1 is a T-cell factor (TCF)/LEF family member. LEF-1 is the main downstream regulator of the Wnt signaling pathway and belongs to the high mobility group (HMG) box family [8]. LEF-1 is involved in regulating the cell cycle and cell proliferation-related genes, such as cyclin D1 and c-myc $[9,10]$. Groucho /TLE can bind two domains of LEF-1 in an HDAC-dependent manner and can competitively inhibit $\beta$ catenin/LEF-1 complex activity [11]. Previous studies indicated that SIRT-1 could inhibit the expression of MMP-13 in human chondrocytes by regulating LEF-1 and has a crucial chondroprotective effect [12]. However, the interaction between SIRT1 and LEF1 in the OA has not yet been fully explained. Hence, it was the main focus of the present study.

\section{Materials And Methods}

\section{Specimen}

Fresh tibial plateau specimens were taken from patients undergoing total knee arthroplasty, and informed consent was obtained. The diagnostic criteria for osteoarthritis established by the American Rheumatology Society were adopted, excluding rheumatoid arthritis, hormonal arthritis and other diseases.

Animals were purchased from Beijing WeitongLihua Laboratory Animal Technology Co., Ltd. (Beijing, China). The mice are kept at $22^{\circ} \mathrm{C}$ and $60 \pm 5 \%$ humidity, free to move around and get food and water, and was provided with a 12-hour light/dark cycle. Sixty female C57BL/6j mice, 8 weeks old (about 20g), were selected and divided into six groups: a 2-week Modeling group (instability of the medial meniscus $n=10$, model $2 w)$, a 2-week SRT1720 treatment group ( $n=10$, SRT 2 w), a 2-week normal group $(n=10$,sham $2 w)$, a 8-week Modeling group ( $n=10$,model $8 w)$, a 8-week SRT 1720 treatment group $(n=10, S R T$ 8w) and a 8 weeks normal group ( $n=10$,sham $8 w)$. The surgical site was the mouse's right knee joint. All operations were destabilizing the medial meniscus $₫ \mathrm{DMM} \otimes$ \operations[13].

\section{SRT1720 drug treatment}

Drug SRT1720 (a specific activator of SIRT1, APEX-bio) was dissolved in dimethyl sulfoxide (DMSO) at $38 \mathrm{mg} / \mathrm{ml}$, and was stored at $-20^{\circ} \mathrm{C}$. SRT 1720 was first diluted in phosphate-buffered saline (PBS), and was administered intraperitoneally twice a week at a dose of $2.5 \mathrm{mg} / 100 \mathrm{~g}$. The drug treatment was started from day $1^{\text {st }}$ after the operation, and later on, the mice were sacrificed after 2 or 8 weeks of treatment using $\mathrm{CO}_{2}$ anesthesia. The fresh specimens of the right knee joint were obtained. The drug dosage and configuration of SRT1720 were adopted from previous studies [14].

\section{Histological evaluation of articular cartilage}


Histological evaluation was performed on Fresh Human tibial plateau specimen, and knee joint specimens were obtained from 6 mice in each group. Specimens of the knee joint were immediately placed in $4 \%$ paraformaldehyde solution and were fixed for 48 hours. The specimens were washed with PBS ( $\mathrm{PH}=7.3)$, and the fluid was changed every hour for 6 hours. For decalcification, 10\% EDTA solution was added for 2 weeks with a change of fluid once a day. Finally, the specimens were embedded in paraffin. Before observation, the specimens were cut into 4 um sagittal sections and were stained with Safranin O Fast Green and Hematoxylin-Eosin. The specimens (damage to Human tibial plateau specimen and mouse) were evaluated according to the OARSI scale developed by the International Osteoarthritis Research Association (OARSI, Table 1).

\section{Immunohistochemistry and immunofluorescence}

For immunohistochemical experiments, paraffin sections were first dewaxed and dehydrated in xylene using $0.1 \%$ trypsin to repair the antigen at $37^{\circ} \mathrm{C}$ for 15 minutes and was then incubated in $3 \%$ hydrogen peroxide solution for 15 minutes to inhibit peroxidase activity. The sections were blocked with Goat serum for 30 minutes and then incubated with the primary antibody overnight at $4^{\circ} \mathrm{C}$. Primary antibodies

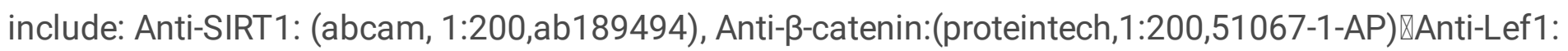
(proteintech,1:200,14972-1-AP),Anti-MMP13:(abcam,1:300,ab39012), Anti-collagen II: (abcam,1:300,ab34712). The number of positive cells was counted with Image pro-plus 6.0 software. For immunofluorescence experiments, the sections were fixed in 4\% PFA and then combined with the corresponding antibody at $4^{\circ} \mathrm{C}$ overnight, followed by a combination with Alexa Fluor 488 goat anti-rabbit secondary antibody (Abcam, 1:300, ab150077) and incubated at $37^{\circ} \mathrm{C}$ for 60 minutes.

An inverted fluorescence microscope was used to observe the positive cells, and Imagepro-plus 6.0 software was used to count the proportion of positive cells.

\section{RNA extraction and quantitative qRT-PCR}

The Quantitative Real-time PCR(qRT-PCR) reaction analysis was performed according to the information specified in the real-time quantitative PCR (MIQE) guidelines issued in 2009. The total RNA was extracted using the tissue RNA extraction kit (AXYGEN, Wujiang, China). Each group comprised of 4 mouse knee cartilage. Total RNA (500ng) was used to prepare cDNA (Bio-rad, Hercules, CA, USA). 25ng cDNA and SYBER Green mixture (TransgenBiotech, Beijing, China) used for the real-time quantitative PCR reaction.

The primer sequence used are as follow:

ß-actin: Forward(F): 5'-GTGCTATGTTGCTCTAGACTTCG-3'

Reverse(R): 5'-ATGCCACAGGATTCCATACC-3'

SIRT-1: (F):5'-AGTAACAGTGACAGTGGCACATGC3/,

(R):5'-GCCTCTCCGTATCATCTTCCAAGC-3/ 
ß-catenin:(F):5'-AGGAATGAAGGCGTGGCAACATAC-3/

(R):5'-GGCACCAATGTCACGTCCAAGATC-3'

LEF-1:(F):5'-GGCGGCGTTGGACAGATCAC-3'

(R):5'-GGATGAGGGATGCCAGTTGTGTG-3 '.

$\beta$-actin was used as a reference housekeeping gene. Relative expression of the target gene was determined using $2-\triangle \triangle \mathrm{CT}$. equation

\section{Statistical analysis}

One-way analysis of variance (One-Way ANOVA) method was used for comparison between multiple groups of data, and the Tukey's multiple comparisons test was used for homogeneity of variance, and the data were expressed as mean \pm SD. $P<0.05$ was considered statistically significant. GraphPad Prism 8 software for statistical analysis.

\section{Results}

\section{The expression of LEF-1 in cartilage tissue varies at different OA stages}

To evaluate the cartilage degeneration of different specimens, we performed safranin 0 fast green staining on fresh human tibial plateau specimens and used the OARSI score to evaluate cartilage degeneration (Fig. 1). The scores of OA mild group, OA moderate group, and OA severe group gradually increased, and the degree of cartilage destruction progressively severe. The cartilage joint surface in the OA mild group was complete, smooth, and continuous; the cartilage joint surface in the OA moderate group was cracked, and chondrocyte hypertrophy; the cartilage in the OA severe group was severely worn, and the subchondral bone was remodeled.

To evaluate the expression of LEF-1 protein in cartilage tissues of different OA stages, we performed immunohistochemistry to detect the expression of LEF-1 protein. The expression of LEF-1 protein in OA mild group, OA moderate group, and OA severe group gradually increased with the severity of cartilage damage, suggesting that LEF-1 protein expression was affected during the development of OA.

\section{SIRT-1 can regulate the expression of LEF-1 protein and play a chondroprotective effect}

To evaluate the effect of SIRT-1 on the protection of osteoarthritis cartilage, we stained animal tissue sections with Safranin O Fast Green and assessed articular cartilage degeneration using OARSI score (Fig. 2). The mice's articular surface in the 2-week normal group and the 8-week normal group was complete, smooth, and continuous, and the cartilage thickness was moderate. Among them, the thickness of the cartilage in the 8-week normal group became slightly thinner. The cartilage surface of the 2 and 8 weeks model mice was discontinuous, and the number decreased. The 8 week model mice lost hyaline 
cartilage and exposed calcified cartilage. The OARSI score and histological analysis showed that compared with the normal group, the scores of model mice at 2 and 8 weeks increased significantly, and the cartilage surface was severely damaged. Compared with the model mice, the cartilage destruction of the mice in the 2-week SRT treatment group and the 8-week SRT treatment group were fewer, and their OARSI scores were also reduced.

To evaluate the specific effects of SIRT-1 on cartilage degeneration, we used immunohistochemical techniques to detect SIRT-1 and LEF-1 proteins (Fig. 2). The expression of SIRT-1 in the model mice at 2 and 8 weeks after surgery decreased, while the expression of LEF- 1 increased than the mice in the normal group. Compared with the model group, the expression of SIRT-1 was increased in the SRT treatment group of 2 and 8 weeks, while the expression of LEF-1 was decreased. In summary, these findings indicate that SIRT-1 and LEF-1 expression are inversely proportional when OA occurs, and SIRT-1 can reduce cartilage degeneration when $\mathrm{OA}$ occurs.

\section{SIRT-1 can reduce the expression of $\beta$-catenin and MMP-13 protein and reduce the degradation of Collagen II}

This research conducted immunohistochemistry experiments to study the regulatory relationship between SIRT-1 and $\beta$-catenin, MMP-13 and Collagen II and its chondroprotective effects (Fig. 3). $\beta$-catenin is a key upstream factor in the Wnt signaling pathway[15]. MMP-13 is the main degradation enzyme of type II collagen in cartilage[16]. Compared with the 2-week normal group and the 8-week normal group, the expressions of $\beta$-catenin and MMP-13 in the 2-week model and the 8-week model groups were increased, the Collagen II protein expression was reduced, the cartilage layer became thinner, and the cartilage cells were significantly less. Compared with the model group, the expressions of $\beta$-catenin and MMP-13, hypertrophy, and death of chondrocytes in the 2-week SRT treatment group and the 8-week SRT treatment group were reduced, and the cartilage layer was thickened.

\section{$\beta$-catenin can reduce the expression of LEF-1 and MMP-13 protein and play a chondroprotective effect}

To study the protective effect of SIRT-1 on cartilage, this research performed immunofluorescence experiments to detect the expression of $\beta$-catenin, LEF-1, and MMP-13 proteins (Fig. 4). Type II collagen is the main collagen component in the extracellular matrix of chondrocytes and is closely related to the occurrence and progression of OA[17]. Compared with the 2-week normal group and the 8-week normal group, the cartilage $\beta$-catenin, LEF-1, and MMP-13 protein expressions in the 2-week and 8-week models were increased, the cartilage layer became thinner and degenerated, and chondrocytes became hypertrophic and necrotic, and the extracellular matrix is reduced. Compared with the model, the expression of $\beta$-catenin, LEF-1, and MMP-13 in cartilage in the 2-week SRT treatment group and 8-week SRT treatment group decreased, the cartilage layer was thickened, the number of cartilage cells increased, and the extracellular matrix increased.

\section{Chondroprotective effects of $\beta$-catenin and inhibition of LEF-1 protein expression}


To study the relationship between $\beta$-catenin and LEF-1, a real-time quantitative polymerase chain reaction (qRT-PCR) experiment was performed. The results showed that compared to the normal group, expression of $\beta$-catenin and LEF-1 mRNA in the model group (2-week and 8-week groups) was significantly high. On the contrary, the expression of $\beta$-catenin and LEF-1 mRNA in the SRT treatment group (2-week and 8-week groups) was significantly reduced compared to the model group.

\section{Discussion}

This study collected fresh tibial plateau specimens from patients with OA. Comparing the OA moderate group and OA severe group, this study find that the cartilage damage in the OA severe group is more serious, the subchondral bone is exposed, and the corresponding LEF-1 protein expression elevated, suggesting that it may play a key role in the occurrence of OA.

SRT-1720 is a small molecule activator, which can specifically increase the expression level of SIRT-1, regulate the energy metabolism efficiency of body tissues, and prevent metabolic disorders. It has been proven to delay the progression of OA [18]. Previous studies have shown that SIRT-1 plays an important role in regulating cell growth, proliferation, and inflammation[19, 20], and meanwhile, it can play a chondroprotective effect by regulating the Wnt/ $\beta$-catenin pathway [21]. When OA occurs, the Wnt signaling pathway is activated, and the Wnt ligand binds to the Frizzled receptor and low-density lipoprotein receptor-related protein $5 / 6$ (LRP5/6) on the plasma membrane, causing the $\beta$-catenin protein to accumulate and translocate to the nucleus. It combines with the N-terminal domain of LEF-1 to form dimers [22, 23], which regulate the expression, proliferation, and migration of downstream genes. This research is the first pre-clinical study to report that SIRT-1 can affect the expression of MMP-13 and Collagen II by regulating the LEF-1, thereby playing a chondroprotective effect.

This study used medial meniscus instability surgery (DMM) to induce OA in mice. This surgery is widely used in the study of OA in mice and can also simulate human OA. It has also been reported that another method of modeling-anterior cruciate ligament transection (ACLT), the development of OA is too fast, so this method is not used. In this experiment, 2 weeks after DMM surgery, mouse cartilage showed early cartilage degeneration, 8 weeks after DMM surgery, cartilage degeneration was more obvious, cartilage was severely damaged, and its thickness became thinner. The OARSI score of the model is much higher than the normal group.

Lymphocyte enhancer factor (LEF-1) exists in the nucleus of mammalian cells. It can combine with $\beta$ catenin and DNA to form a ternary compound, regulating the bending and transcription of the corresponding DNA[22]. In the mice model, this study have observed that as the severity of OA increases, the expression of $\beta$-catenin and LEF-1 increase simultaneously, and cartilage thickness increases, confirming that the chondroprotective effect of SIRT-1 may be through $\beta$-catenin/LEF-1 Shaft to achieve. Studies have shown that $\beta$-catenin and HDAC1 act in a LEF-1-dependent manner,compared with unbound HDAC1, the combined activity of HDAC1 is reduced [23]. In mice lacking LEF-1, this study have observed 
that the skin, teeth, hair, etc., are missing, and their life span is significantly affected, suggesting that LEF1 plays an important role in the growth and development of organisms [24].

As a nicotinamide adenine dinucleotide $\left(\mathrm{NAD}^{+}\right)$-dependent histone deacetylase, SIRT-1 plays a key role in regulating many signal transduction pathways such as cell aging, apoptosis, autophagy, and repair of DNA damage $[25,26]$, SIRT-1 protein is expressed in the nucleus of all cartilage and synovial tissues and has the ability to deacetylate which is associated with a variety of age-related diseases [27]. It has been found that the expression of HDAC2 and HDAC7 is upregulated in OA cartilage. The use of HDAC inhibitors can inhibit the degradation of cartilage and extracellular matrix in cartilage $[5,28,29]$. Moreover, as a natural HDAC inhibitor, trichostatin (TSA) can increase the ratio of matrix metalloproteinase inhibitor - 1(TIMP-1)/matrix metalloproteinase (MMP) in OA mice, which has a chondroprotective effect [30]. Previous studies show that HDAC inhibitors play a protective role in OA, which may be another exciting research direction in this field.

\section{Conclusion}

The preventive treatment of OA reflects the protective treatment of its articular cartilage. This research proves that SIRT-1 regulates downstream factors through the LEF-1 to play a chondroprotective effect. It provides new ideas for the prevention and treatment of OA.

\section{List Of Abbreviations}

Silent information regulator 1, SIRT-1; lymphocyte enhancer factor 1, LEF-1; Matrix metalloproteinase 13, MMP-13; Osteoarthritis, OA; A Disintegrin And Metalloproteinase With Thrombospondin ,ADAMTS; Histone deacetylase, HDAC; T-cell factor, TCF; High mobility group, HMG; Destabilizing the medial meniscus, DMM; Dimethyl sulfoxide, DMSO; Phosphate-buffered saline, PBS; Osteoarthritis Research Society International, OARSI; Quantitative Real-time PCR, qRT-PCR; Low-density lipoprotein receptorrelated protein 5/6,LRP5/6; Anterior cruciate ligament transection, ACLT; Nicotinamide adenine dinucleotide, NAD ${ }^{+}$; Trichostatin, TSA; Metallopeptidase inhibitor 1, TIMP1;

\section{Declarations}

\section{Availability of data and materials}

All the data will be available upon motivated request to the corresponding author of the present paper.

\section{Ethics approval and consent to participate}

All experiments were approved by the Animal Experiment Ethics Committee of Ningxia Medical University (protocol number: 2019-066) and approved by the Medical Research Ethics Review Committee of the General Hospital of Ningxia Medical University (Proposal Number: 2020-999). All experiments were 
carried out under the standard principles of animal experiment ethics and comply with the Declaration of Helsinki.

\section{Consent for publication}

Written informed consent was obtained from each patient to authorize the publication of their data.

\section{Declaration of interest}

The authors declare that they have no competing interests

\section{Funding}

This research was supported by the scientific research project of Ningxia Medical University (XT2020008) and the scientific research project of General Hospital of Ningxia Medical University (020007004127).

\section{Author contribution}

Qunhua Jin and Long Ma designed this experiment, and Xueyu Hu, Gangning Feng, Zhiqiang Meng conducted the main experiment. Xueyu Hu and ZhiQiang Meng analyzed the main data and drafted the manuscript to modify it. All authors read and approved the final manuscript

\section{References}

1. Thomas Hu Gle A J G:What drives osteoarthritis-synovial versus subchondral bone pathology.Rheumatology,2017;9:1461-71

2. Correa D L S A:Articular cartilage repair: Current needs, methods and research directions.Seminars in Cell \& Developmental Biology,2017;2:67-77

3. Richardson A M B:Articular cartilage: structure, injuries and review of management.Br Med Bull,2008;1:77-95

4. Goldring M B G:Articular cartilage and subchondral bone in the pathogenesis of osteoarthritis.Annanls of the new york academy of sciences,2010;1:230-37

5. Lomeli R. Carpio J J W:Histone Deacetylases in Cartilage Homeostasis and Osteoarthritis.Curr Rheumatol Rep,2016;8:1-9

6. Abed É C D D:Low sirtuin 1 levels in human osteoarthritis subchondral osteoblasts lead to abnormal sclerostin expression which decreases Wnt/ $\beta$-catenin activity.Bone,2014;28-36

7. Matsushita T SHT:The overexpression of SIRT1 inhibited osteoarthritic gene expression changes induced by interleukin-1 $\beta$ in human chondrocytes.Journal of Orthopaedic Research,2013;4:531-37

8. Santiago L D G W:Wnt signaling pathway protein LEF1 in cancer, as a biomarker for prognosis and a target for treatment.American journal of cancer research,2017;6:1389-1406 
9. Micheal Shtutman J Z I S:The cyclin D1 gene is a target of the beta-catenin/LEF-1 pathway.Proceedings of the National Academy of Sciences,1999;9610:5522-27

10. Tong-Chuan He A B S C:Identification of C-MYC as a target of the APC pathway.Science (New York, N.Y.),1998;5382:1509-12

11. Laura Arce K T P:Groucho binds two conserved regions of LEF-1 for HDAC-dependent repression.BMC cancer,2009;1:1-14

12. Elayyan J L E J:LEF1-mediated MMP13 gene expression is repressed by SIRT1 in human chondrocytes. The FASEB Journal,2017;7:3116-25

13. Glasson S B T M:The surgical destabilization of the medial meniscus (DMM) model of osteoarthritis in the 129/SvEv mouse.Osteoarthritis and Cartilage,2007;9:1061-69

14. K. Nishida T M K T:Intraperitoneal injection of the sIRT1 activator SRT1720 attenuates the progression of experimental osteoarthritis in mice.Bone Joint Reserch,2018;3:252-62

15. Lietman C W B L,Rossomacha E:Inhibition of Wnt/ $\beta$-catenin signaling ameliorates osteoarthritis in a murine model of experimental osteoarthritis.JCl insight,2018;3:1-16

16. Meina Wang E R S H:MMP13 is a critical target gene during the progression of osteoarthritis.Arthritis Res Ther,2013;1:1-11

17. Ali Mobasheri C L Y H:Coll2-1 and Coll2-1NO2 as exemplars of collagen extracellular matrix turnover - biomarkers to facilitate the treatment of osteoarthritis?Expert Rev Mol Diagn,2019;9:803-12

18. Je Ro^ Me N. Feige M L C C:Specific SIRT1 activation mimics low energy levels and protects against diet-induced metabolic disorders by enhancing fat oxidation.Cell Metab,2008;5:347-58

19. Hiroyasu Yamamoto K S:Sirtuin functions in health and disease.Molecular endocrinology,2007;8:1745-55

20. Jae-Suk Seoa M M J J:SIRT1, a histone deacetylase, regulates prion protein-induced neuronal cell death.Neurobiology of aging,2012;6:1110-20

21. Shuan Liu HY B H:Sirt1 regulates apoptosis and extracellular matrix degradation in resveratrol-treated osteoarthritis chondrocytes via the $\mathrm{Wnt} / \beta$-catenin signaling pathways.Experimental And Therapeutic Medicine,2017;5:5057-62

22. Jurgen Behrens J P V K:Functional interaction of b-catenin with the transcription factor LEF1.Nature,1996;6592:638-42

23. Cara Jamieson M S B R:Targeting the $\beta$-catenin nuclear transport pathway in cancer.Seminars in Cancer Biology,2014;4:1-10

24. Courtney Van Genderen R M O I:Development of several oreans that require inductive epithelialmesenchymal interactions is impaired in LEF-1- deficient mice.Gene and Development,1994;822:2691-2703

25. Li X:SIRT1 is a Cellular Metabolic Sensor.Acta Biochim. Biophys. Sin.,2012;45:51-60

26. Rabiyacoub K L A J:The Role of SIRT1 in Diabetic Kidney Disease.Frontiers in endocrinology,2014;166:1-8 
27. Gabay O C K A:Epigenetics of cartilage diseases.Joint Bone Spine,2016;5:491-94

28. Jingwei Lu Y S Q G:Histone deacetylase 4 alters cartilage homeostasis in human osteoarthritis.BMC musculoskeletal disorders,2014;1-8

29. Choi G I Y:Epigenetics in osteoarthritis and its implication for future therapeutics. Expert opinion on biological therapy,2013;5:713-21

30. Hao Qu J L L W:Trichostatin A increases the TIMP-1/MMP ratio to protect against osteoarthritis in an animal model of the disease.Molecular Medicine Reports,2016;143:2423-30

\section{Table}

Table 1: OA cartilage histopathology grade assessment-degrading methodology

\begin{tabular}{|c|c|}
\hline Grade (key feature) & Associated criteria (tissue reaction) \\
\hline $\begin{array}{c}\text { Grade 0: surface intact, cartilage } \\
\text { morphology intact }\end{array}$ & $\begin{array}{l}\text { Matrix: normal architecture } \\
\text { Cells: intact, appropriate orientation }\end{array}$ \\
\hline Grade 1: surface intact & $\begin{array}{l}\text { Matrix: superficial zone intact, oedema and/or superficial fibrillation (abrasion)focal } \\
\text { superficial matrix condensation } \\
\text { Cells: death, proliferation (clusters), hypertrophy, superficial zone Reaction must be more } \\
\text { than superficial fibrillation only }\end{array}$ \\
\hline Grade 2: surface discontinuity & $\begin{array}{l}\text { As above }+ \text { matrix discontinuity at superficial, zone (deep fibrillation) } \pm \text { Cationic stain matrix } \\
\text { depletion (Safranin O or Toluidine Blue) upper } 1 / 3 \text { of cartilage } \pm \text { focal } \\
\text { perichondronal increased stain (mid zone) } \pm \text { disorientation of chondron columns } \\
\text { Cells: death, proliferation (clusters), hypertrophy }\end{array}$ \\
\hline Grade 3: vertical fissures (clefts) & $\begin{array}{l}\text { As above }+ \text { matrix vertical fissures into mid zone, branched fissures } \pm \text { cationic stain } \\
\text { depletion (Safranin } O \text { or Toluidine Blue) into lower } 2 / 3 \text { of cartilage (deep zone) } \pm \text { New } \\
\text { collagen formation (polarized lightmicroscopy, Picro Sirius Red stain) } \\
\text { Cells: death, regeneration (clusters), hypertrophy, cartilage domains adjacent to fissures }\end{array}$ \\
\hline Grade 4: erosion & $\begin{array}{l}\text { Cartilage matrix loss: delamination of superficial layer, mid layer cyst formation. } \\
\text { Excavation: matrix loss superficial layer and mid zone }\end{array}$ \\
\hline Grade 5: denudation & $\begin{array}{l}\text { Surface: sclerotic bone or reparative tissue including fibrocartilage within denuded surface. } \\
\text { Microfracture with repair limited to bone surface }\end{array}$ \\
\hline Grade 6: deformation & $\begin{array}{l}\text { Bone remodelling (more than osteophyte formation only). Includes: microfracture formation } \\
\text { only). Includes: microfracture with fibrocartilaginous and osseous repair extending } \\
\text { above the previous surface }\end{array}$ \\
\hline
\end{tabular}

\section{Figures}


OA mild
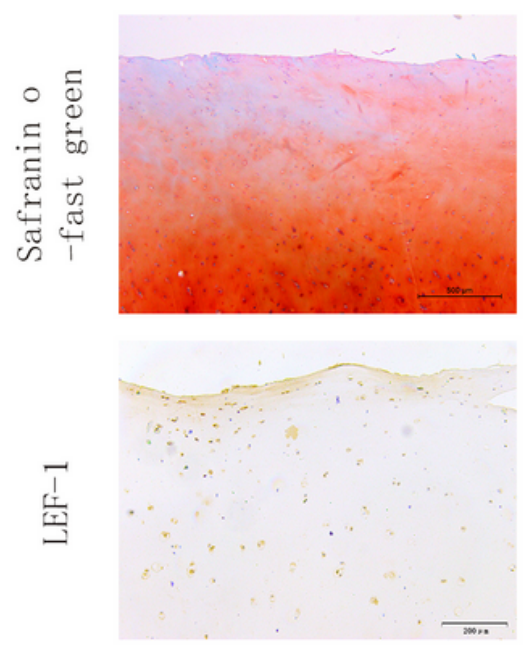

OA moderate
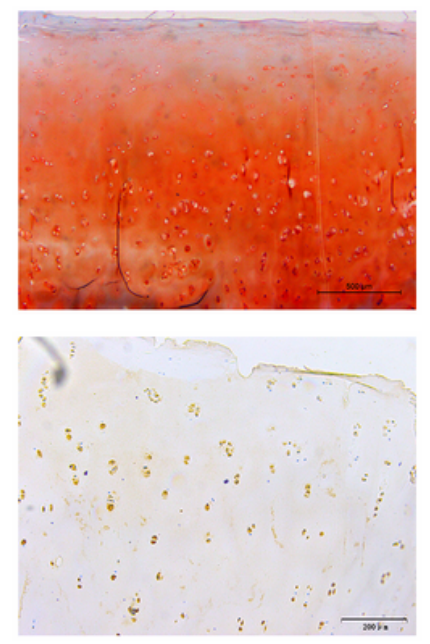

OA severe
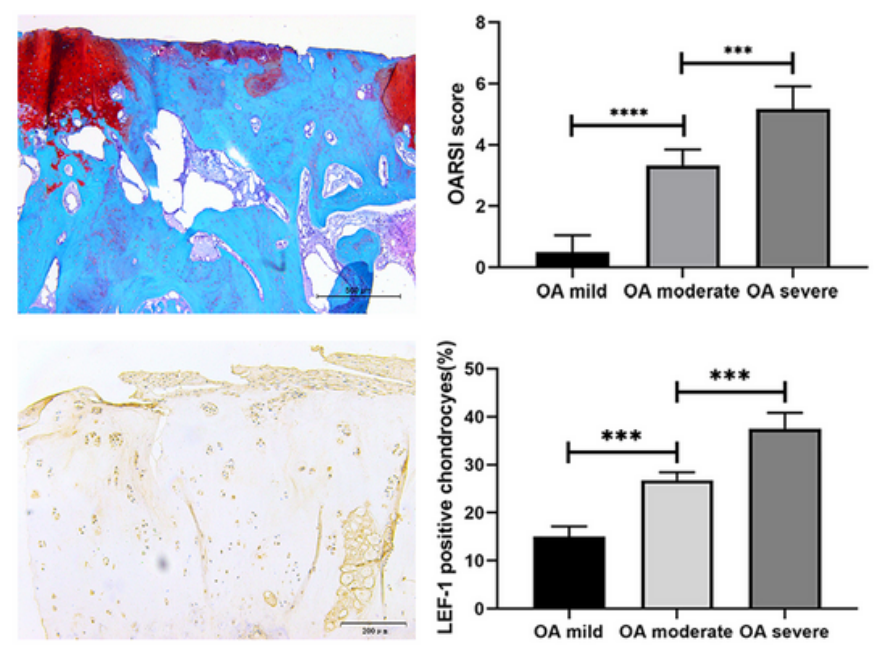

\section{Figure 1}

The expression of LEF-1 protein in cartilage of different degrees of damage is different. Safranin 0 fast green staining was used to process fresh tibial plateau tissue sections, and the OARSI score issued by the International Association of Osteoarthropathy was used to evaluate the degree of cartilage degeneration. The use of immunohistochemical techniques to detect the expression of LEF-1 in the specimen. Safranin $O$ fast green staining scale bar: $500 \mu \mathrm{m}$, LEF-1 scale bar: $200 \mu \mathrm{m}$. The OA severe group vs the OA moderate group, ${ }^{\star \star *}, \mathrm{P} \otimes 0.001$. 

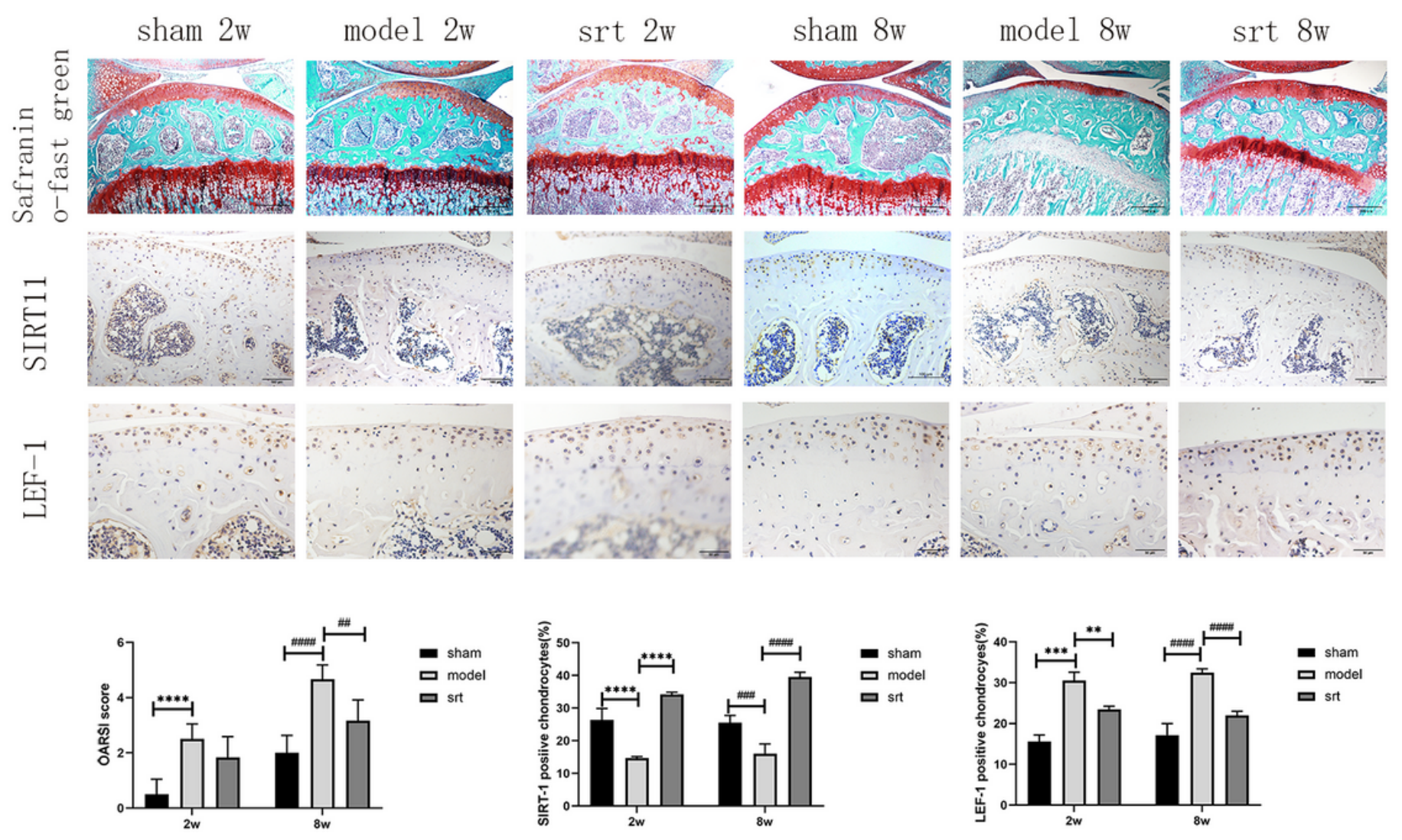

\section{Figure 2}

SIRT1 can regulate the expression of LEF-1 to reduce cartilage damage. Safranin 0 fast green staining was used to process mouse knee joint cartilage sections, and the OARSI score issued by the International Osteoarthropathy Association was used to assess the degree of cartilage degeneration. Use immunohistochemical technology to detect the expression of SIRT-1 and LEF-1. Safranin 0 fast green staining scale bar: $200 \mu \mathrm{m}$, SIRT-1 and LEF-1 scale bar: 50 $\mu \mathrm{m}$. 2-week group: normal group vs model mice, $\star \star \star \star, ~ P<0.0001 ;$ 8-week group: model mice vs SRT treatment group, \#\#, $\mathrm{P}<0.01$. 


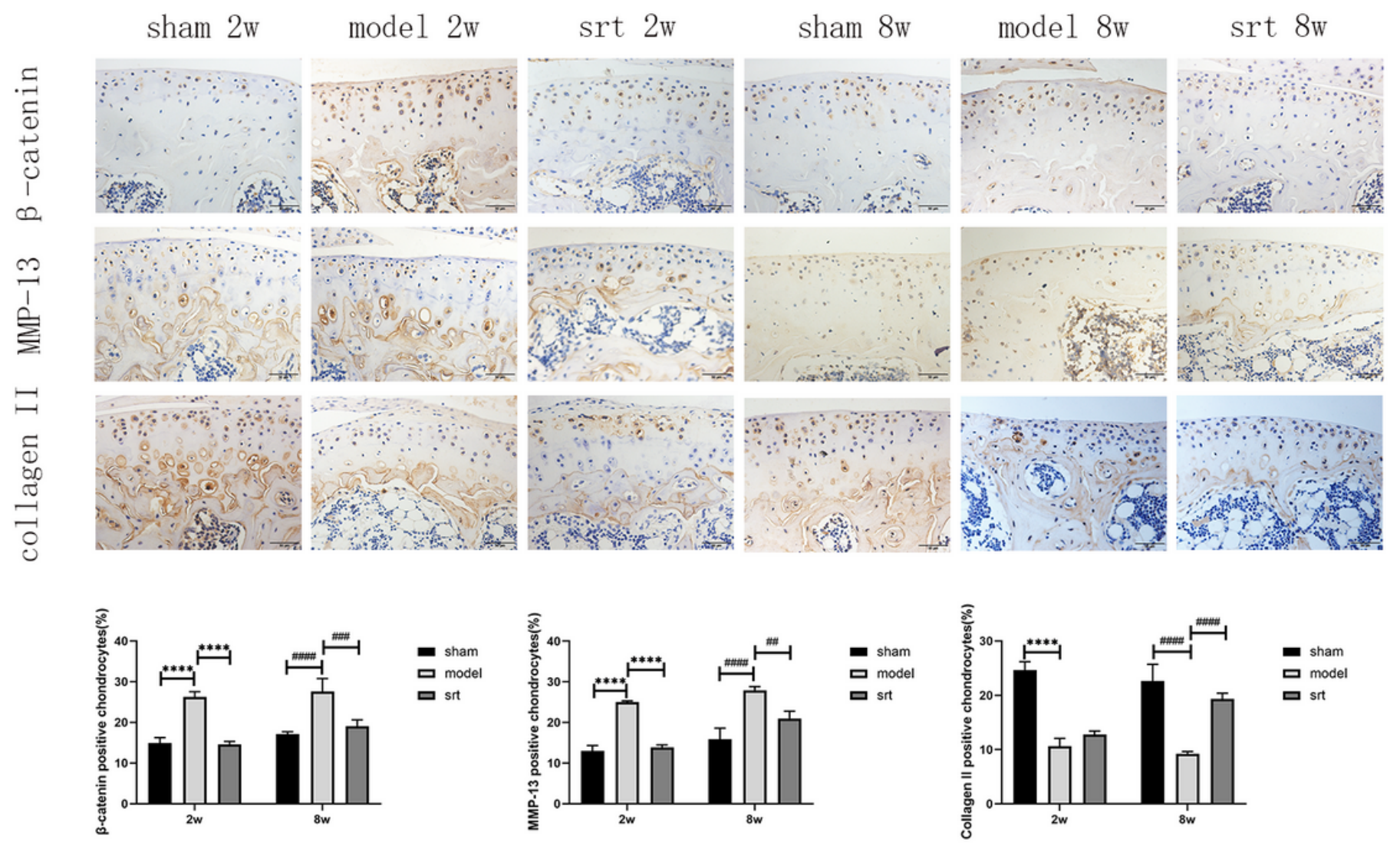

Figure 3

SIRT-1 can reduce $\beta$-catenin and MMP-13 protein expression and reduce Collagen II protein degradation. . Immunohistochemistry was used to detect the expression of $\beta$-catenin, MMP-13 and Collagen II proteins. $\beta$-catenin, MMP-13 and Collagen II scale bar: $50 \mu \mathrm{m}$. 2-week group: normal group vs model mice, ${ }^{\star \star \star \star}$, $\mathrm{P}<0.0001 ;$ 8-week group: model mice vs SRT treatment group, \#\#\#, $\mathrm{P}<0.001$. 
sham 2 w
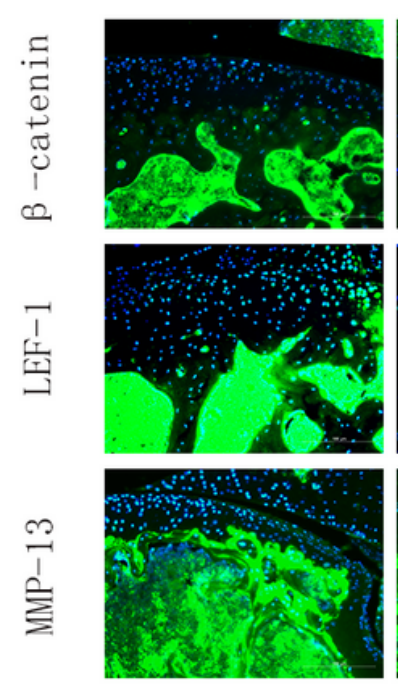

model $2 \mathrm{w}$
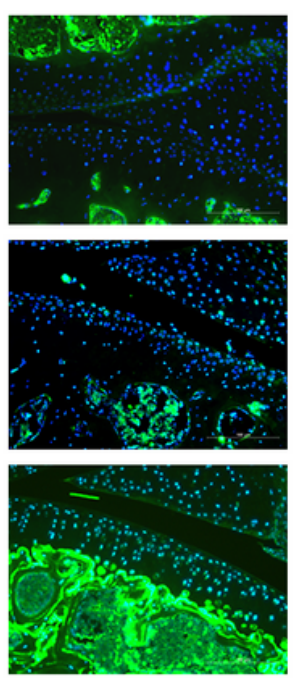

$\operatorname{srt} 2 \mathrm{w}$
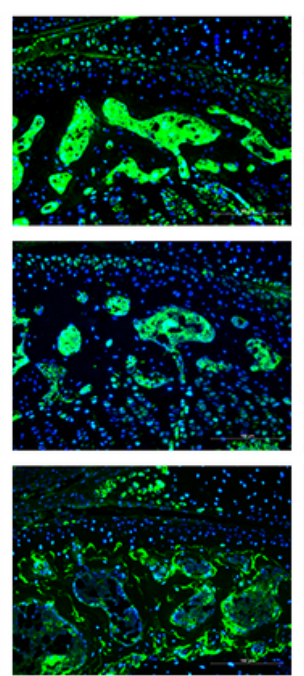

sham $8 w$
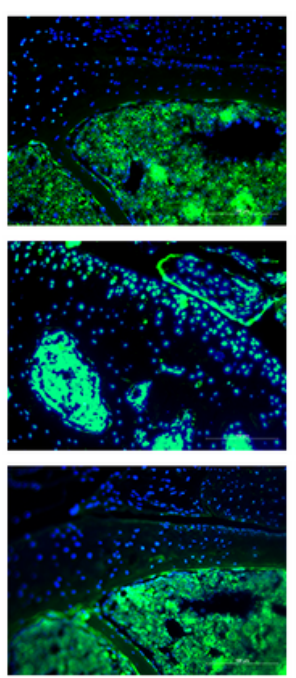

model 8w
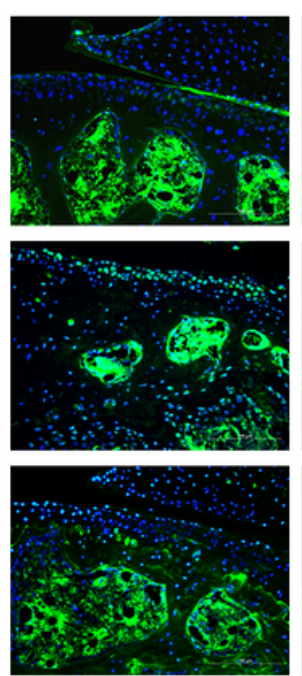

srt $8 w$
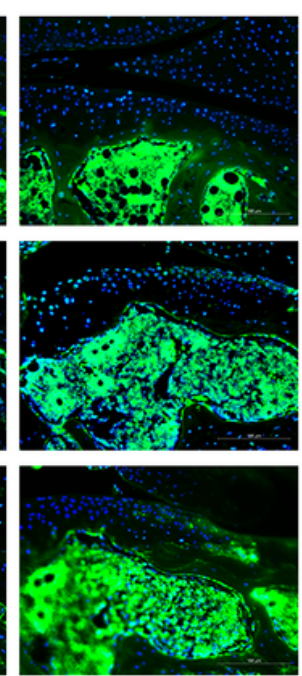
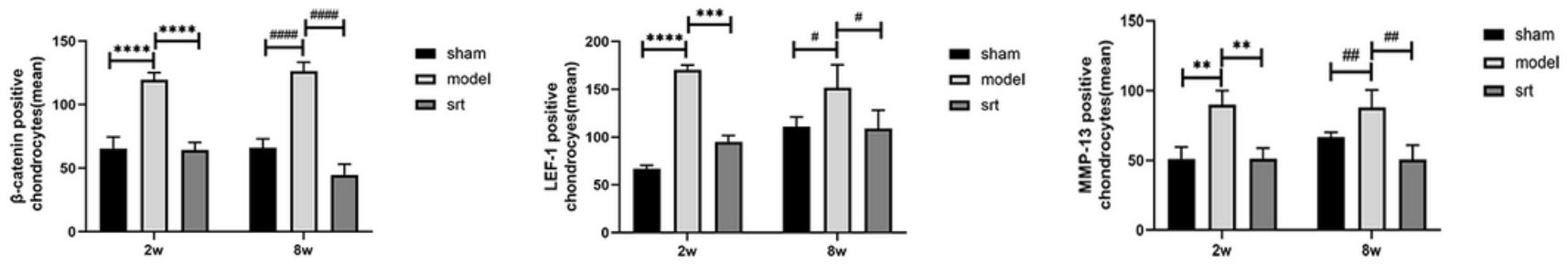

Figure 4

LEF-1 can regulate the metabolism of type II collagen to play a chondroprotective effect. Immunofluorescence technology was used to detect the expression of $\beta$-catenin, LEF-1 and MMP-13 protein. $\beta$-catenin, LEF-1 and MMP-13 scale bar: 100 $\mu \mathrm{m}$. 2 weeks group: normal group vs model mice, $\star \star \star \star, ~ P<0.0001 ; 8$ week group: model mice vs SRT treatment group, \#\#\#\#, P<0.0001.
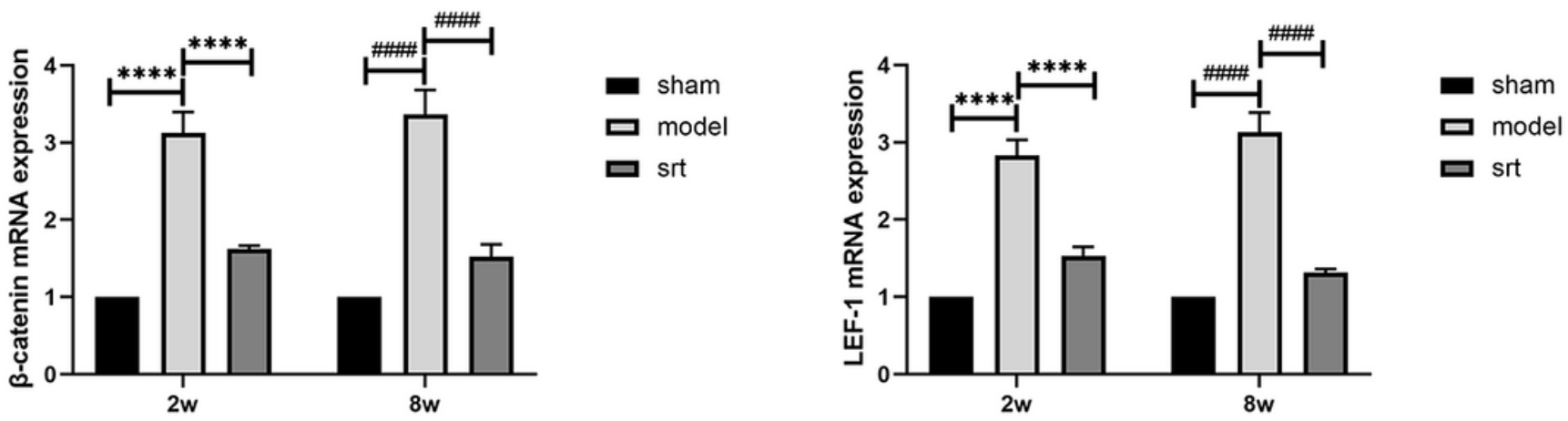

Figure 5

$\beta$-catenin reduces the expression of LEF-1 protein and plays a chondroprotective effect. RT-qpcr technology was used to detect the mRNA expression of $\beta$-catenin, LEF-1 and MMP13. 2-week group: 
normal group vs model mice, $\star \star \star \star, ~ P<0.0001 ; 8$-week group: model mice vs SRT treatment group, \#\#, $\mathrm{P}<0.01$. 